\title{
Compassion toward others and self-compassion predict mental and physical well-being: a 5-year longitudinal study of 1090 community-dwelling adults across the lifespan
}

\author{
Ellen E. Lee $\mathbb{D}^{1,2,3 凶}$, Tushara Govind ${ }^{3}$, Marina Ramsey ${ }^{1,3}{ }^{1,3}$ Tsung Chin Wu ${ }^{4}$, Rebecca Daly ${ }^{1,3}$, Jinyuan Liu ${ }^{4}$, Xin M. Tu ${ }^{4}$, \\ Martin P. Paulus (D) ${ }^{5}$, Michael L. Thomas ${ }^{6}$ and Dilip V. Jeste ${ }^{1,3,7}$
}

(c) The Author(s) 2021

\begin{abstract}
There is growing interest in the role of compassion in promoting health and well-being, with cross-sectional data showing an inverse correlation with loneliness. This is the first longitudinal study examining both compassion toward others (CTO) and compassion toward self (CTS) as predictors of mental and physical health outcomes including loneliness, across adult lifespan. We followed 552 women and 538 men in San Diego County for up to 7.5 (mean 4.8 and SD 2.2) years, using validated rating scales for CTO, CTS, and loneliness. Linear mixed-effects models were employed to examine age- and sex-related trajectories of CTO and CTS over time. Linear regression models were used to evaluate baseline and longitudinal relationships of CTO and CTS with mental wellbeing, physical well-being, and loneliness. CTS and CTO were weakly intercorrelated. Women had higher baseline CTO than men. While CTO was stable over time and across the lifespan, CTS scores had an inverse U-shaped relationship with age, peaking around age 77. There were significant baseline $\times$ slope interactions of both CTO and CTS predicting improvements in physical well-being in adults $<60$ years old. Increases in CTO and CTS predicted improvements in mental well-being. Higher baseline CTO and CTS as well as increases in CTO and CTS scores predicted lower loneliness scores at follow-up. Thus, CTO and CTS were associated with better mental well-being and loneliness across the adult lifespan, and physical well-being in younger adults, and are promising targets for interventions to improve health outcomes.
\end{abstract}

Translational Psychiatry (2021)11:397; https://doi.org/10.1038/s41398-021-01491-8

\section{INTRODUCTION}

Pro-social attitudes and behaviors like compassion have been linked to greater well-being and better health in individuals and potentially, in the society $[1,2]$. Compassion involves recognizing suffering of others and then taking action to help them [3]. A related construct is empathy, which enables individuals both cognitively and emotionally to identify and experience the mental states of other people, allowing comprehension of and engagement in social relationships [4]. Empathy is necessary for compassion but is not sufficient because the latter also requires motivation followed by action. Compassion is considered to include two subtypes, according to the target of compassion-compassion toward others (CTO) and compassion toward self (CTS) [3, 5].

From an evolutionary perspective, СTO has been purported to support caring for vulnerable offspring (as human infants and children require a much higher level and longer duration of care relative to other mammals), aid mate selection, and increased cooperation between non-related individuals [5]. CTS may also have a beneficial role of tempering self-criticism. Self-criticism can be important for stimulating threat processing although it can also be associated with increased anxiety and depression [6].
Compared to men, women have been reported to have greater levels of affective and cognitive empathy as well as higher CTO [710], but lower CTS [11-17]. From an evolutionary standpoint, only women have childbearing roles, and traditionally they have also had greater caregiving responsibilities. Therefore, higher CTO levels may be associated with female sex both biologically and culturally. The reported relationships of CTO and CTS with age has been mixed, though longitudinal studies are lacking. While crosssectional studies of CTO $[3,9,10]$ and CTS $[16,18,19]$ have reported similar levels among older versus younger adults, several cross-sectional studies of empathy have demonstrated lower levels of cognitive empathy [20-25], but similar levels of affective empathy in older adults [4, 22, 25-28]. The few published longitudinal studies of empathy in older adults have had mixed findings: five with increasing empathy with aging (especially after age 40) [29], one with decline in empathy (especially in women) [30], and one with stable levels of empathy with aging [31]. These longitudinal findings are limited by somewhat varied definitions of empathy, use of different measures, unbalanced sex distributions $[29,30]$, insufficient racial/ethnic/socioeconomic diversity in the sample, and a lack of meaningful health outcomes. Similarly, the

\footnotetext{
${ }^{1}$ Department of Psychiatry, University of California San Diego, La Jolla, CA, USA. ${ }^{2}$ VA San Diego Healthcare System, San Diego, CA, USA. ${ }^{3}$ Sam and Rose Stein Institute for Research on Aging, University of California San Diego, La Jolla, CA, USA. ${ }^{4}$ Department of Family Medicine and Public Health, University of California San Diego, La Jolla, CA, USA. ${ }^{5}$ Laureate Institute for Brain Research, Tulsa, OK, USA. ${ }^{6}$ Department of Psychology, Colorado State University, Fort Collins, CO, USA. ${ }^{7}$ Department of Neurosciences, University of California San Diego, La Jolla, CA, USA. ${ }^{凶}$ email: eel013@health.ucsd.edu
} 
"Grandmother Hypothesis" [32] posits that older women of postreproductive ages support the species through helping raise grandchildren, enhancing fertility of their adult daughters, and survival and longevity of the grandchildren [33]. Thus, from an evolutionary standpoint, CTO and CTS may have important roles across the lifespan and in both sexes, though prior studies have not examined sex- and age-specific changes in CTO and CTS.

During recent decades there has been a behavioral pandemic of loneliness, associated with higher morbidity and all-cause mortality [34, 35]. Loneliness is defined as subjective distress arising from discrepancies between desired and perceived social relationships [36], and has somewhat distinct health consequences from social isolation which refers to an objective lack of social contact and support [34]. The increased mortality from loneliness and associated suicides and opioid-related deaths has led to lowering the average US lifespan two years in a row after a progressive increase since 1959 [37]. Our earlier cross-sectional study suggested three age groups in which loneliness seemed to peak-late 20s, mid-50s, and late 80 s [35]. A consistent finding in four published studies has been a strong inverse correlation between loneliness and wisdom, a multi-component personality trait, and its main component-pro-social behaviors of empathy and compassion [35, 38-40]. A recent study using EEG also provided neurobiological support to the negative association between these traits [41]. Another study found that loneliness and wisdom, especially its compassion component, were associated with lower versus higher, respectively, levels of alpha and beta diversity of gut microbial taxa composition [42].

Cross-sectional studies have reported associations of compassion with better mental health (greater happiness and well-being [43-45]) and physical health (lower cardiovascular risk [2] and decreased inflammation [46]). However, to our knowledge, the interrelationship of CTO and CTS, how CTO and CTS change across the adult lifespan, as well as their possible association with mental (including loneliness) and physical health have not been examined longitudinally in sizable cohorts of women and men. Pro-social attitudes and behaviors are partially modifiable [47-54] and should be an excellent potential target for healthfocused interventions across the adult lifespan. Interventions to enhance CTO and CTS have been shown to improve health outcomes [47-54]. For example, two studies reported that a Mindfulness Self-Compassion Program improved CTS and also reduced diabetes distress and hemoglobin A1C in adults with diabetes $[47,55]$. While promising, the published interventions have usually not examined the associations with age and sex, had relatively short follow-up periods of only a few months, and did not assess both CTO and CTS using validated measures. The longer-term sex- and age-related associations between CTO, CTS, and health are unknown. It is not clear what are the relative importance of the relationship of CTO and CTS to physical and mental health, including loneliness.

In the current study, CTO and CTS were assessed in a relatively large sex-balanced community-based sample across the adult lifespan (age range 27-101 years) that was followed longitudinally for up to 7.5 (mean 4.8 and SD 2.2) years. We sought to examine: (1) the relationship between CTO and CTS; (2) sex differences in CTO and CTS; (3) how CTO and CTS vary by sex across the lifespan; and (4) the relationship of CTO and CTS to physical and mental health including loneliness. We hypothesized that (1) baseline CTO and CTS levels would be highly intercorrelated, and (2) women would have higher levels of CTO than men. We explored (3) how CTO and CTS would change longitudinally by sex and across the age-span. Finally, we hypothesized that (4) higher baseline levels and longitudinal increases in CTO and CTS would predict higher levels of mental well-being including lower loneliness, as well as better physical health at the end of the follow-up period, controlling for smoking, alcohol use, and relevant sociodemographic factors.

\section{METHODS}

\section{Study participants}

The study participants were recruited from the UCSD Successful AGing Evaluation (SAGE) study across the adult lifespan, which has been described previously $[56,57]$. Briefly, participants were recruited using list-assisted random digit dialing in San Diego county. The inclusion criteria were (1) community-dwelling adults, (2) aged 21-100+ years, (3) provision of written informed consent to participate in the study, (4) fluency in English, (5) a telephone line at home, (6) physical and mental abilities to complete the study assessments, and (7) no known diagnosis of dementia. Persons who lived in nursing homes or required daily skilled nursing care, or had a terminal illness, were excluded. All participants included in the current study had CTO and CTS assessments and annual follow-up data available (two or more data-points).

The study protocol was approved by the UC San Diego Human Research Protections Program (HRPP) and all the participants provided a written informed consent prior to study participation. The data were collected over a period from July 2012 through February 2020.

\section{Sociodemographic and clinical characteristics}

Trained study staff completed a 25 -min initial phone interview followed by a survey that was mailed or completed online. Data included sociodemographic information on age, sex, education level, race/ethnicity, current marital status, living situation, and income. Self-administered standardized assessments were completed for depression (Patient Health Questionnaire-9 or PHQ-9) [58], anxiety (Brief Symptom Inventory - Anxiety subscale) [59], perceived stress (Perceived Stress Scale) [60], resilience (Connor Davidson Resilience Scale or CD-RISC) [61], optimism (Life Orientation Test - Revised or LOTR) [62], and satisfaction with life (Satisfaction with Life Scale or SWLS) [63]. Loneliness was measured only at the end of the study, with the 20-item UCLA-3 Loneliness Scale [64]. Physical and mental health assessments included mental and physical well-being based on the composite scores from the Medical Outcomes Survey-Short Form 36 (SF-36) [65].

CTO was assessed with the Santa Clara Brief Compassion Scale, a 5-item scale with items rated with 7-point Likert scale (1: "Not at all true of me" to 7: "Very true of $\mathrm{me}^{\prime \prime}$ ) [8]. This validated measure was based on the 21-item Sprecher and Fehr Compassionate Love for Humanity Scale [7], and is highly correlated with the original version $(r=0.96)$ with high internal reliability (Cronbach's alpha of 0.90 ). An example item is "I tend to feel compassion for people, even though I do not know them." To measure CTS, we used the Neff Self-compassion Scale short-form, a 12-item adaptation of the popular and well-validated 25-item scale [66], that has strong correlation to the original version $(r \geq 0.97)$ and strong internal consistency (Cronbach's alpha $\geq 0.86$ ). The items are rated on a 5-point Likert scale (1: "Almost never" to 5: "Almost always"). An illustrative item is "When I'm going through a very hard time, I give myself the caring and tenderness I need."

The participants were followed longitudinally for up to 7 years with assessments being performed at Years 0 (baseline), 1, 2, 3, 6, and 7.

\section{Statistical analyses}

Independent sample $t$-tests and chi-square tests were used to assess differences in sociodemographic factors and various outcome measures between male and female participants. Log transformation was used for non-normally distributed variables. To address Question 1, the baseline relationship between CTO and CTS was assessed using Spearman's correlation. For Question 2, we used independent sample $t$-tests to compare CTO and CTS by sex. For Question 3, linear mixed-effects models were used to determine trajectories of CTO and CTS over time (polynomial), with age, sex, marital status, race, and household income at baseline as the predictors, along with interactions of time with each of the demographic variables. Two random-effects structures were chosen with one including a random intercept only and the other including both a random intercept and random slope. The optimal structure was selected by using REML AIC [67]. Sandwich variance estimates were used to improve inference validity.

For Question 4, multiple linear regression models were developed to examine associations of the individual baseline and slope values of CTO and CTS calculated based on the baseline and last observed outcomes model with changes in mental and physical health. Inference was based on estimating equations to improve inference validity [68]. To account for moderation effect by baseline CTO and CTS, the model also included baseline by slope interaction for CTO and CTS. All models controlled for 
age, sex, marital status, race, and household income at baseline [69]. Due to a previously reported beginning of a sharp decline in physical wellbeing around age 60 in our sample [63], longitudinal models of physical well-being were run separately for individuals $\leq 60$ years old and those $>60$ years. The regression model was also used to assess associations of CTO and CTS with loneliness at follow-up. The analyses were repeated by adding smoking and alcohol use at baseline as additional covariates.

We present effect sizes and $p$-values for all of these statistical tests, and interpret small-medium effect sizes (i.e., Cohen's $d>0.20$ or $r \geq 0.30$ ) as meaningful. Statistical significance was defined as alpha $=0.05$ (two-tailed) for all analyses.

\section{RESULTS}

\section{Sample characteristics}

Our sample consisted of comparable numbers of women $(N=538)$ and men $(N=552)$. The men were significantly older, had more education and higher household income, and were less likely to be non-Caucasian or in a marriage-like relationship (Table 1). Women had significantly higher anxiety, but depression levels were similar between the sexes.

\section{Question 1: Intercorrelation of CTO and CTS at baseline}

CTO and CTS were weakly correlated to each other $(r=0.16, p \leq$ 0.001 in the whole sample; $r=0.09, p=0.02$ in women; $r=0.20$, $p<0.001$ in men). CTS and CTO were more strongly intercorrelated within men $(z=-1.99, p=0.02)$.

\section{Question 2: Baseline sex differences in CTO and CTS}

Baseline CTS scores did not differ significantly by sex; however, women had significantly higher CTO than men (5.0 \pm 1.2 vs. $4.4 \pm$ $1.4, t_{1,064}=6.93, p<0.001, d=0.42$ ).

\section{Question 3: Longitudinal relationships of compassion with sex} and age

Figure 1 shows changes in CTO and CTS plotted against follow-up year and against baseline age, based on results from the linear mixed-effects model. Women had significantly higher CTO than men throughout the follow-up period and across all age groups $\left(B=-0.56, \mathrm{SE}=0.07, t_{1,065}=-7.86, p \leq 0.001\right)$ (Fig. 1A). Follow-up year had a non-linear association with CTO $(B=0.006, \mathrm{SE}=0.001$, $\left.t_{3,156}=5.06, p \leq 0.001\right)$, though 7-year changes in CTO were modest across age groups. Non-Caucasian individuals had higher CTO levels than Caucasians, and lifetime abstainers had higher CTO levels than current regular drinkers. Baseline age, marital status, income, smoking, and infrequent/former drinking were not related to changes in CTO.

Sex and follow-up year were not significantly related to CTS levels $\left(B=0.28, \mathrm{SE}=0.42, t_{1,041}=0.67, p=0.50\right.$; and $B=0.02$, $\mathrm{SE}=0.04, t_{3,029}=0.48, p=0.63$; respectively). Baseline age had a cubic association with CTS $\left(B=-0.0007, \mathrm{SE}=<0.001, t_{1,041}=\right.$ $-2.87, p=0.004)$, with peak CTS at age 77. At baseline age in the $40 \mathrm{~s}$ and $60 \mathrm{~s}$, CTS increased slightly throughout the follow-up period, while it remained stable among participants in their $20 \mathrm{~s}$ and 90s (Fig. 1B). Annual household income $>\$ 35,000$ was associated with higher CTS. Marital status, race, smoking, and alcohol use were not related to changes in CTS.

\section{Question 4a: Longitudinal associations of compassion with physical and mental well-being}

The models of physical well-being differed by age group. In the younger age group ( $\leq 60$ years old), baseline values as well as changes in CTO and CTS were associated with changes in physical well-being (Table $2 A)$. There was a significant interaction between baseline CTO and slope of CTO $\left(B=-0.74, \mathrm{SE}=0.25, \eta_{\mathrm{p}}{ }^{2}=0.025\right)$, such that individuals with higher baseline CTO had less robust CTO-related increases in physical well-being. Conversely, individuals with lower baseline CTO had more robust CTO-related increases in physical well-being. There was also a significant baseline CTS $\times$ slope of CTS interaction $(B=0.03, \mathrm{SE}=0.009$, $\left.\eta_{\mathrm{p}}{ }^{2}=0.041\right)$, such that individuals with lower baseline CTS had less robust CTS-related increases in physical well-being. The effect sizes for the CTO and CTS variables in predicting physical wellbeing were greater than those of smoking and drinking alcohol. The model that included CTO and CTS had a better model-fit compared to the model without CTO and CTS [goodness-of-fit metrics (QIC) were 1919 and 2126, respectively].

In the older group (>60 years old), baseline and changes in CTS and CTO were not significantly associated with changes in physical well-being (Table 2B).

Greater increases in CTO and CTS predicted improvements in mental well-being (Table 2C). There was no significant interaction between baseline scores and subsequent changes in CTO or CTS scores.

\section{Question 4b: Longitudinal association of compassion with loneliness}

Higher baseline CTO and CTS, as well as greater increases in СTO and CTS scores significantly predicted lower loneliness scores at the last follow-up (Table 3, with small effect sizes for CTO and medium effect sizes for CTS). Being male, being single at baseline, and having annual household incomes $<\$ 75,000$ were associated with higher loneliness scores at the last follow-up (with small effect sizes). Infrequent drinkers, regular drinkers, and former drinkers all had significantly lower loneliness scores than lifetime abstainers. Race, age, and smoking habits did not have a significant relationship to loneliness.

\section{DISCUSSION}

The current study aimed to examine the baseline and longitudinal sex- and age-related associations of CTO and CTS with mental and physical health, and loneliness. Our findings show weak correlation between CTO and CTS, clear sex differences in baseline levels as well as longitudinal trajectories of CTO, and an inverse- $U$ association of CTS with age, and significant associations of CTO and CTS with physical well-being in younger adults as well as with mental well-being and loneliness across the lifespan. Overall, these findings partially support our hypotheses. While CTO and CTS are not highly intercorrelated, CTO and CTS are closely and independently linked to health and loneliness, though sex and age appear to influence those associations.

While, to our knowledge, there have been no multi-year longitudinal follow-up studies of CTO and CTS across the adult lifespan, we did find a few published longitudinal studies examining changes in empathy with aging [29-31, 70-73]. Five longitudinal studies reported increases in empathy with aging $[29,70-73]$, while two found either no change or a small linear decline in empathy $[30,31]$. The current findings of an inverted-Ushaped relationship between age and CTS are similar to those of O'Brien and colleagues from a sample of adults age 18-90 years, with middle-aged adults having greater affective and cognitive empathy than younger and older adults [74]. Grühn and colleagues found cross-sectional age differences but no changes longitudinally, and attributed their findings to cohort differences in empathy levels in a sample aged 15-87 years. Researchers have attributed changes in empathy to social networking use, media and technology consumption, and changes in other psychological characteristics and behaviors, parenting, and family practices, and the broader cultural zeitgeist (e.g., a culture of success or a culture of social consciousness) [29]. In this study, the decline in CTS after age 77 may reflect age-related declines in cognitive empathy and social cognition or generational differences [31, 74], though this study did not explicitly examine social cognition measures. For example, the middle-aged group in this sample had formative experiences, such as coming of age during the Civil Rights Movement, that may have influenced their development of CTS. 
Table 1. Baseline demographic and clinical comparison of women and men in the study sample.

\begin{tabular}{|c|c|c|c|c|c|c|c|c|c|c|}
\hline & \multicolumn{3}{|c|}{ Women } & \multicolumn{3}{|l|}{ Men } & \multirow[b]{2}{*}{$t$ or $X^{2}$} & \multirow[b]{2}{*}{ df } & \multirow[b]{2}{*}{$p$} & \multirow[b]{2}{*}{ Cohen's $d$} \\
\hline & $N$ & $\begin{array}{l}\text { Mean } \\
\text { or } \%\end{array}$ & SD & $N$ & $\begin{array}{l}\text { Mean } \\
\text { or \% }\end{array}$ & SD & & & & \\
\hline \multicolumn{11}{|l|}{ Sociodemographic } \\
\hline Age at baseline visit (years) & 552 & 61.5 & 22.0 & 538 & 66.0 & 20.3 & -3.51 & 1088 & $<0.001$ & -0.21 \\
\hline Race (\% Caucasian) & 403 & 73.1 & & 421 & 78.5 & & 4.33 & 1 & 0.04 & \\
\hline Education (\%) & & & & & & & 20.0 & 1 & $<0.001$ & \\
\hline Post-graduate degree & 99 & 18.2 & & 157 & 29.4 & & & & & \\
\hline Household income (\%) & & & & & & & 14.2 & 2 & 0.001 & \\
\hline$<\$ 35,000$ & 143 & 28.9 & & 100 & 19.8 & & & & & \\
\hline$\$ 35,000-\$ 74,999$ & 161 & 32.5 & & 160 & 31.7 & & & & & \\
\hline Alcohol use & & & & & & & 20.0 & 3 & $<0.001$ & \\
\hline Lifetime abstainers & 84 & 15.9 & & 66 & 12.6 & & & & & \\
\hline Infrequent drinkers & 290 & 55.0 & & 241 & 45.9 & & & & & \\
\hline Regular drinkers & 90 & 17.1 & & 144 & 27.4 & & & & & \\
\hline Former drinkers & 63 & 12.0 & & 74 & 14.1 & & & & & \\
\hline \multicolumn{11}{|l|}{ Psychopathology } \\
\hline Anxiety (BSIA) & 541 & 2.1 & 3.3 & 529 & 1.5 & 2.4 & 2.98 & 1068 & 0.003 & 0.18 \\
\hline Depression (CESD) & 529 & 5.4 & 4.6 & 526 & 5.0 & 4.4 & 1.65 & 1053 & 0.10 & 0.10 \\
\hline \multicolumn{11}{|l|}{ Compassion measures } \\
\hline CTS (Neff Scale) & 531 & 41.4 & 8.0 & 515 & 42.5 & 7.2 & -2.22 & 1044 & 0.03 & -0.14 \\
\hline
\end{tabular}

BSIA Brief Symptom Inventory - Anxiety subscale, CESD Center for Epidemiologic Studies Depression Scale, CTO compassion toward others, CTS Compassion toward self, SCBCS Santa Clara Brief Compassion Scale, SF-36 36-item Short Form Survey, UCLA UCLA Loneliness Scale [assessed only at follow-up].

Thus, both aging and cohort effects are important to consider for CTO and CTS.

Cross-sectional studies have reported higher empathy among women on both cognitive and affective empathy measures [20, 74-79]. Schieman and Van Gundy reported that the gap between the sexes closed in older ages [20]. Women have also been reported to have higher CTO than men across a number of cross-sectional studies in community-dwelling populations [7$10,80]$, healthcare professionals [81], and younger adults [82]. as well as lower CTS than men in a meta-analysis [17]. Most of the longitudinal studies found higher empathy levels among women compared to men in their samples [29, 70-73] with one report of no sex difference across the lifespan [31]. Some of these longitudinal samples lacked sex balance, especially in older ages $[29,30]$, a wide range of socioeconomic status, and racial/ethnic diversity-though these appear to be key covariates of CTO and CTS.

While the current study found higher annual income $(>\$ 35,000)$ to be associated with higher CTS but not CTO, the literature linking CTO/CTS to education and socioeconomic status (SES) is complex and mixed. Cross-sectional studies reported that individuals with more education and higher SES had lower CTO
[83] and higher CTS levels [84], which may be linked to the communal advantage of pro-social behaviors in communities with low SES [85]. Conversely, higher SES may reduce reliance on others and result in lower CTO [86], though the decreased parental stress and increased parental support in high SES environments during childhood may promote pro-social behaviors [87]. A 32-year longitudinal study reported that high childhood SES predicted higher СTO at age 30-40 and higher adulthood CTO was linked to higher adulthood SES at 10-year follow-up [87].

CTO and CTS represent distinct constructs. CTO has sometimes been associated with negative outcomes. For instance, one study found that caregivers with higher CTO experienced more intrusive thoughts about their care recipients' condition than caregivers with lower CTO, regardless of the level of physical suffering that they saw in the care recipient. In concordance with the current findings, three observational studies of adults across the lifespan, college undergraduates, and nursing students reported weak or non-significant correlations between CTS and CTO [84, 88, 89]. However, compassion-based interventions may improve both CTO and CTS. A meta-analysis of such interventions (e.g., Compassion Focused Therapy, Mindful Self-Compassion) to enhance CTO or CTS found an overall improvement in both CTO and CTS, as well as 
A Compassion Towards Others

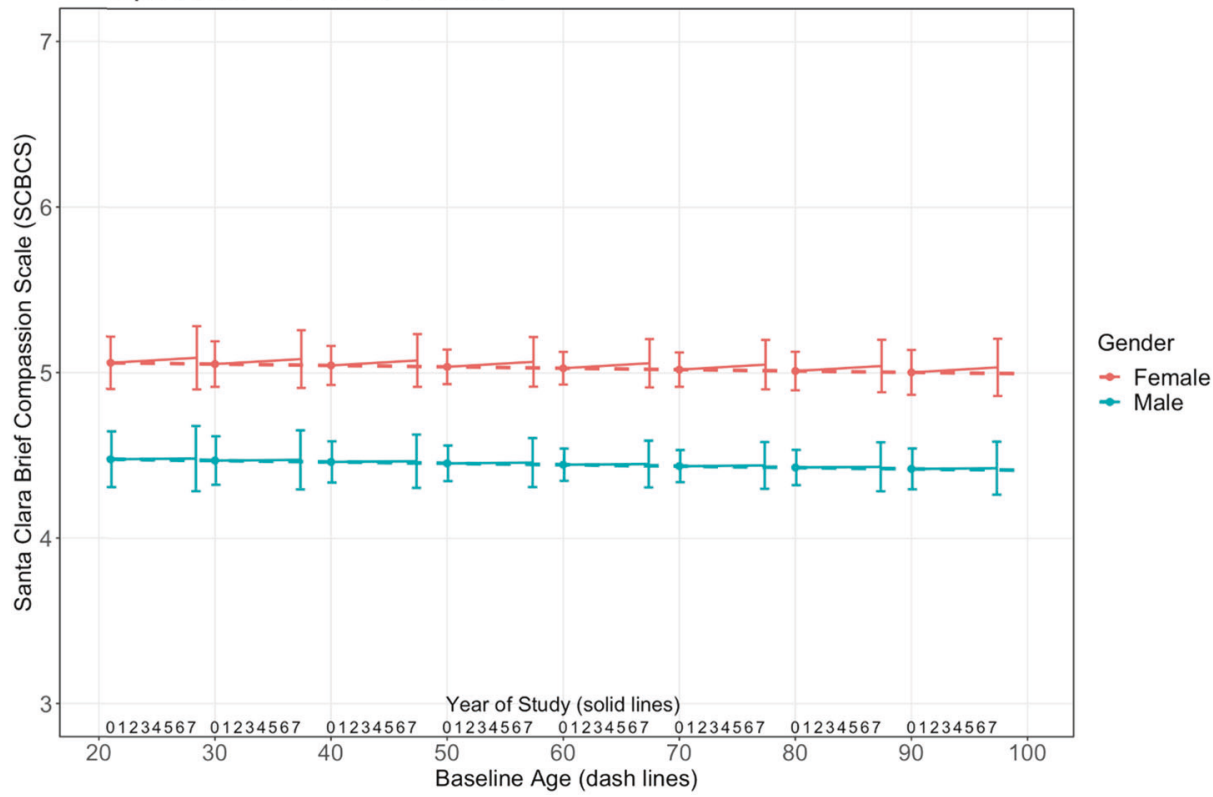

B Compassion Towards Self

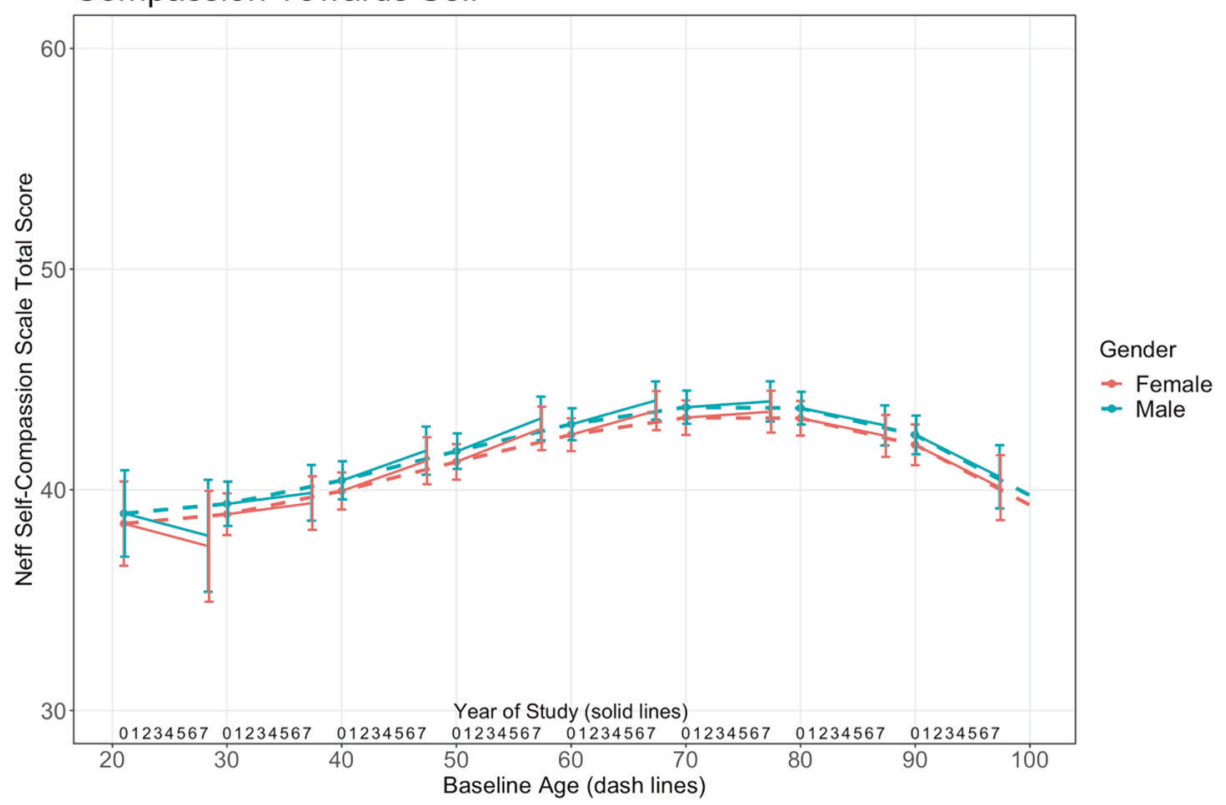

Fig. 1 Trajectories of compassion toward others (CTO) and compassion toward self (CTS) by age and gender. A Trajectories of compassion toward others (CTO) by Age and Gender (Mean follow-up 4.8 years, up to 7 years). Solid lines depict the changes in CTO levels up to 7 years of follow-up by decade of age. Dotted lines show the best-fit model of CTO with baseline age. B Trajectories of compassion toward self (CTS) by age and gender (mean follow-up 4.8 years, up to 7 years). Solid lines depict the changes in CTS levels up to 7 years of follow-up by decade of age. Dotted lines show the best-fit model of CTS with baseline age.

overall well-being [90]. Our results highlight the sex differences in the CTO-CTS correlations, which could reflect baseline sex differences in CTO and CTS or sex-specific influences that affect CTO and CTS levels. These findings warrant further investigations in sex-related contributory factors.

The influence of CTO and CTS on physical health was consistent with prior studies which showed that CTS improved lifestyle behaviors and metabolic biomarkers among adults with diabetes $[47,55,91]$. Reviews have found that CTS interventions can improve health behaviors from goal-setting and monitoring [92] to affective responses [93]. These psychosocial determinants of health are important factors to consider for improving health and well-being across the lifespan. However, a case can also be made for reverse causality, i.e., individuals with better mental and physical health have greater CTO and CTS. The current study's longitudinal findings appear to support a ceiling effect with CTO, such that individuals with higher CTO at baseline did not have CTO-related improvement in physical health. On the other hand, the longitudinal findings appear to have a floor effect with CTS, such that individuals with lower CTS at baseline did not have CTSrelated improvement in physical health. Furthermore, CTO and CTS were not linked to physical health in adults $60+$ years of age, 
Table 2. Multiple linear regression models of compassion toward others (CTO) and compassion toward self (CTS) with the outcomes of physical well-being among individuals age $\leq 60$ years (A) outcomes of physical well-being among individuals age $>60$ years (B), and mental wellbeing (C).

\section{Prediction of change in physical well-being over 5 years

$\begin{array}{lllll}B & S E & \text { Wald } & p & \eta_{p}{ }^{2}\end{array}$

(A) Baseline and changes in compassion toward others (CTO) and compassion toward self (CTS) as predictors of change in physical wellbeing over 7 -year follow-up in individuals age $\leq 60$ years

\begin{tabular}{|c|c|c|c|c|c|}
\hline Intercept & 0.44 & 0.91 & 0.23 & 0.63 & \\
\hline Baseline СТО & 0.13 & 0.09 & 1.91 & 0.17 & 0.002 \\
\hline Slope CTO & 3.19 & 1.02 & 9.77 & 0.002 & $<0.001$ \\
\hline Baseline CTS & -0.03 & 0.02 & 2.29 & 0.13 & 0.006 \\
\hline Slope CTS & -1.25 & 0.38 & 10.71 & 0.001 & 0.05 \\
\hline Age & -0.01 & 0.01 & 1.25 & 0.26 & 0.004 \\
\hline Gender (men) ${ }^{\mathrm{a}}$ & 0.28 & 0.25 & 1.32 & 0.25 & 0.004 \\
\hline $\begin{array}{l}\text { Marital status } \\
\text { (marriage-like) }^{\mathrm{b}}\end{array}$ & -0.01 & 0.30 & $<0.01$ & 0.96 & $<0.001$ \\
\hline Race (Caucasian) ${ }^{c}$ & 0.05 & 0.26 & 0.03 & 0.86 & $<0.001$ \\
\hline Household income $^{d}$ & & & & & 0.01 \\
\hline$\$ 35,000-\$ 74,999$ & -0.56 & 0.47 & 1.39 & 0.24 & \\
\hline$\geq \$ 75,000$ & 0.02 & 0.36 & $<0.01$ & 0.95 & \\
\hline Smoking (ever) ${ }^{\mathrm{e}}$ & 0.002 & 0.31 & $<0.01$ & 0.99 & $<0.001$ \\
\hline Alcohol use ${ }^{f}$ & & & & & 0.006 \\
\hline Infrequent drinkers & 0.36 & 0.30 & 1.40 & 0.24 & \\
\hline Regular drinkers & 0.09 & 0.40 & 0.05 & 0.83 & \\
\hline Former drinkers & 0.62 & 0.52 & 1.39 & 0.24 & \\
\hline $\begin{array}{l}\text { Baseline } \\
\text { CTO*Slope CTO }\end{array}$ & -0.74 & 0.25 & 8.99 & 0.003 & 0.025 \\
\hline $\begin{array}{l}\text { Baseline } \\
\text { CTS*Slope CTS }\end{array}$ & 0.03 & 0.009 & 8.13 & 0.004 & 0.041 \\
\hline
\end{tabular}

(B) Baseline and changes in compassion toward others (CTO) and compassion toward self (CTS) as predictors of change in PHysical Wellbeing over 7-year follow-up in individuals age $>60$ years

\begin{tabular}{|c|c|c|c|c|c|}
\hline Intercept & -1.23 & 2.05 & 0.36 & 0.55 & \\
\hline Baseline CTO & -0.25 & 0.15 & 2.99 & 0.08 & 0.007 \\
\hline Slope CTO & 0.79 & 0.94 & 0.71 & 0.40 & 0.006 \\
\hline Baseline CTS & -0.02 & 0.03 & 0.36 & 0.55 & 0.001 \\
\hline Slope CTS & 0.31 & 0.26 & 1.43 & 0.23 & 0.031 \\
\hline Age & 0.03 & 0.02 & 1.43 & 0.23 & 0.004 \\
\hline Gender (men) ${ }^{\mathrm{a}}$ & -0.32 & 0.36 & 0.83 & 0.36 & 0.002 \\
\hline $\begin{array}{l}\text { Marital status } \\
\text { (marriage-like) }^{\mathrm{b}}\end{array}$ & 0.66 & 0.43 & 2.37 & 0.12 & 0.006 \\
\hline Race (Caucasian) $^{c}$ & 0.26 & 0.38 & 0.48 & 0.48 & 0.001 \\
\hline Household income $^{d}$ & & & & & 0.011 \\
\hline$\$ 35,000-\$ 74,999$ & -0.77 & 0.54 & 2.03 & 0.15 & \\
\hline$\geq \$ 75,000$ & -0.01 & 0.55 & $<0.01$ & 0.98 & \\
\hline Smoking (ever) & 0.41 & 0.35 & 1.38 & 0.24 & 0.003 \\
\hline Alcohol use ${ }^{f}$ & & & & & 0.001 \\
\hline Infrequent drinkers & 0.10 & 0.53 & 0.03 & 0.86 & \\
\hline Regular drinkers & 0.07 & 0.55 & 0.01 & 0.90 & \\
\hline Former drinkers & -0.13 & 0.73 & 0.03 & 0.86 & \\
\hline
\end{tabular}

Table 2 continued

\section{Prediction of change in physical well-being over 5 years}

$B \quad$ SE Wald $p \quad \boldsymbol{\eta}_{\mathrm{p}}{ }^{2}$

(C) Baseline and changes in compassion toward others (CTO) and compassion toward self (CTS) as predictors of change in mental wellbeing over 7 years

\begin{tabular}{|c|c|c|c|c|c|}
\hline Intercept & -0.05 & 0.12 & 0.18 & 0.68 & \\
\hline Baseline СТO & 0.01 & 0.01 & 0.98 & 0.32 & 0.002 \\
\hline Slope CTO & 0.13 & 0.03 & 4.37 & 0.04 & 0.02 \\
\hline Baseline CTS & -0.001 & 0.002 & $<0.01$ & 0.95 & $<0.001$ \\
\hline Slope CTS & 0.05 & 0.02 & 6.46 & 0.01 & 0.098 \\
\hline Age & -0.001 & 0.001 & 1.92 & 0.17 & 0.004 \\
\hline Gender (men) ${ }^{a}$ & 0.02 & 0.03 & 0.24 & 0.63 & 0.001 \\
\hline $\begin{array}{l}\text { Marital status } \\
\text { (marriage-like) }^{\mathrm{b}}\end{array}$ & -0.01 & 0.04 & 0.11 & 0.74 & $<0.001$ \\
\hline Race (Caucasian) $^{c}$ & 0.03 & 0.03 & 1.08 & 0.30 & 0.001 \\
\hline Household income $^{d}$ & & & & & 0.003 \\
\hline$\$ 35,000-\$ 74,999$ & -0.001 & 0.04 & $<0.01$ & 0.98 & \\
\hline$\geq \$ 75,000$ & 0.04 & 0.04 & 0.78 & 0.38 & \\
\hline
\end{tabular}

CTO compassion toward others, CTS compassion toward self.

${ }^{a}$ Compared to women, ${ }^{b}$ compared to single, 'compared to non-Caucasian, ${ }^{d}$ compared to $<\$ 35,000$, ${ }^{e}$ compared to never, ${ }^{f}$ compared to lifetime abstainers.

Bold values indicates statistically significant $p$ values $<0.05$.

Table 3. Best-fit linear model of compassion toward others (CTO) and compassion toward self (CTS) with loneliness at follow-up.

\begin{tabular}{|c|c|c|c|c|c|}
\hline & \multicolumn{5}{|c|}{ Loneliness at follow-up } \\
\hline & B & SE & Wald & $p$ & $\eta_{p}^{2}$ \\
\hline Intercept & 76.13 & 3.12 & 594.81 & $<0.001$ & \\
\hline Baseline CTO & -0.76 & 0.31 & 6.19 & 0.01 & 0.01 \\
\hline Slope CTO & -5.36 & 2.26 & 5.62 & 0.02 & 0.01 \\
\hline Baseline CTS & -0.66 & 0.05 & 152.53 & $<0.001$ & 0.22 \\
\hline Slope CTS & -3.34 & 0.44 & 56.49 & $<0.001$ & 0.13 \\
\hline Age & -0.04 & 0.02 & 2.60 & 0.11 & 0.005 \\
\hline Gender (men) ${ }^{\mathrm{a}}$ & 2.01 & 0.76 & 6.92 & 0.009 & 0.01 \\
\hline $\begin{array}{l}\text { Marital status } \\
\text { (marriage-like) }^{\mathrm{b}}\end{array}$ & -3.47 & 0.84 & 17.09 & $<0.001$ & 0.03 \\
\hline Race (Caucasian ${ }^{c}$ & 0.14 & 0.86 & 0.02 & 0.87 & $<0.001$ \\
\hline Household income $^{d}$ & & & & & 0.01 \\
\hline$\$ 35,000-\$ 74,999$ & -1.38 & 1.14 & 1.46 & 0.23 & \\
\hline$\geq \$ 75,000$ & -2.64 & 1.18 & 4.97 & 0.03 & \\
\hline Smoking (ever) ${ }^{\mathrm{e}}$ & -0.59 & 0.76 & 0.61 & 0.44 & 0.001 \\
\hline Alcohol use ${ }^{f}$ & & & & & 0.01 \\
\hline Infrequent drinkers & -2.78 & 1.13 & 6.08 & 0.01 & \\
\hline Regular drinkers & -3.54 & 1.27 & 7.75 & 0.005 & \\
\hline Former drinkers & -3.29 & 1.47 & 4.99 & 0.03 & \\
\hline
\end{tabular}

СТО compassion toward others, CTS compassion toward self.

${ }^{a}$ Compared to men, ${ }^{b}$ compared to single, 'compared to non-Caucasian,

dcompared to $<\$ 35,000$, e compared to never, ${ }^{\mathrm{f}}$ compared to lifetime abstainer.

Bold values indicates statistically significant $p$ values $<0.05$. 
likely due to the large number of confounding factors with older age. Randomized controlled trials (RCTs) of CTO and CTS interventions are warranted to specifically examine whether CTO and CTS are causally linked to health.

Our finding of both CTO and CTS being predictors of lower loneliness at follow-up provides support to the previous findings of a significant inverse correlation $(r=0.51-0.76)$ between loneliness and compassion or wisdom in at least four different cross-sectional studies [35, 38-40]. Though further empirical evidence is warranted, compassionate behavior can be a less socially threatening way to connect with others, as it is likely to be well-received and reciprocated [94]. Furthermore, CTO and CTS are reflective of empathic abilities (understanding the emotions and perspectives of others) that may bolster more rewarding social relationships. There is a possible neurobiological basis for a counteracting effect of compassion on loneliness. A recent EEG study found that measures of loneliness and wisdom (especially its compassion component) were related to contrasting modulations of cognitive processes (reduced versus enhanced response speed biased by angry versus happy emotions, respectively) and invoked similar (temporo-parietal junction) and distinct (superior parietal vs. insula, respectively) neural circuits in specific emotional contexts [47].

These findings support a need for broad investigations into the naturalistic development of CTO and CTS and how these traits may vary by cohort and environmental factors (using longitudinal studies in diverse cohorts from the community), biological mechanisms linked to developing CTO and CTS (inclusion of relevant biomarkers in longitudinal studies as well as RCTs of neurobiological compassion-focused interventions such as brain stimulation and biofeedback), and causal links between CTO/CTS and health (RCTs of compassion-based interventions with adequate follow-up periods).

This study has several limitations. The sample was predominantly Caucasian, and the results may not generalize to racial/ ethnic minorities or to non-English-speaking adults. All the clinical assessments were self-report-based and did not include objective measures. We did not evaluate empathy in this study. Also, we did not begin assessing loneliness until the last followup; therefore, the contribution of CTO and CTS to the longitudinal changes in loneliness is not known. Also, we did not examine biomarkers of inflammation or other markers of stress or aging. The maximum longitudinal follow-up period was 7.5 years, and it is possible that longer follow-up might have produced different results.

Nonetheless, the study also has several strengths. To our knowledge, this is the first multi-year longitudinal study of CTO and CTS across the adult lifespan. It was based on a communitybased sample selected using random digit dialing, with comparable numbers of women and men. We used validated rating scales and controlled for various sociodemographic factors.

Future research is warranted to identify the innate personality features and environmental influences (life events, relationships, cultural and societal norms) that may alter CTO and CTS in diverse populations. In that vein, the benefits of CTO and CTS interventions have not been fully characterizedi.e., whether these interventions have lasting effects on health and other outcomes and which individuals benefit most from such interventions. Last, using biomarkers and objective assessments of CTO and CTS can improve our understanding of the biological underpinnings of pro-social behaviors. Efforts to promote compassion at individual and societal levels may help stem the modern behavioral pandemics of loneliness, stress, suicides, and opioid abuse, worsened by the recent Covid-19 pandemic and the necessary social distancing requirements $[95,96]$.

\section{REFERENCES}

1. Smith JL. Self-compassion and resilience in senior living residents. Sr Hous Care J. 2015;23:16-31.

2. Friedberg JP, Suchday S, Srinivas VS. Relationship between forgiveness and psychological and physiological indices in cardiac patients. Int J Behav Med. 2009;16:205-11.

3. Strauss C, Lever Taylor B, Gu J, Kuyken W, Baer R, Jones F, et al. What is compassion and how can we measure it? A review of definitions and measures. Clin Psychol Rev. 2016;47:15-27.

4. Beadle JN, de la Vega CE. Impact of aging on empathy: review of psychological and neural mechanisms. Front Psychiatry. 2019;10:331.

5. Goetz JL, Keltner D, Simon-Thomas E. Compassion: an evolutionary analysis and empirical review. Psychol Bull. 2010;136:351-74.

6. Gilbert P. The origins and nature of compassion focused therapy. Br J Clin Psychol. 2014;53:6-41.

7. Sprecher S, Fehr B. Compassionate love for close others and humanity. J Soc Personal Relatsh. 2005;22:629-51.

8. Hwang JY, Plante T, Lackey K. The development of the Santa Clara brief compassion scale: an abbreviation of Sprecher and Fehr's compassionate love scale. Pastor Psychol. 2008;56:421-8.

9. Martins D, Nicholas N, Shaheen M, Jones L, Norris K. The development and evaluation of a compassion scale. J Health Care Poor Underserved. 2013;24:1235-46.

10. Moore RC, Martin AS, Kaup AR, Thompson WK, Peters ME, Jeste DV, et al. From suffering to caring: a model of differences among older adults in levels of compassion. Int J Geriatr Psychiatry. 2015;30:185-91.

11. Neff KD. The development and validation of a scale to measure self-compassion. Self and Identity. 2003;2:223-50.

12. Neff KD, Hsieh Y-P, Dejitterat K. Self-compassion. Achiev Goals, Coping Academic Fail. 2005;4:263-87.

13. Raes F. Rumination and worry as mediators of the relationship between selfcompassion and depression and anxiety. Pers. Individ. Dif. 2010;48:757-61.

14. Neff KD, McGehee P. Self-compassion and psychological resilience among adolescents and young adults. Self and Identity. 2010;9:225-40.

15. Yarnell LM, Neff KD. Self-compassion, interpersonal conflict resolutions, and wellbeing. Self Identity. 2013;12:146-59.

16. Ferrari M, Yap K, Scott N, Einstein DA, Ciarrochi J. Self-compassion moderates the perfectionism and depression link in both adolescence and adulthood. PLoS ONE. 2018;13:e0192022.

17. Yarnell LM, Stafford RE, Neff KD, Reilly ED, Knox MC, Mullarkey M. Meta-analysis of gender differences in self-compassion. Self Identity. 2015;14:499-520.

18. Benzo RP, Kirsch JL, Nelson C. Compassion, mindfulness, and the happiness of healthcare workers. Explore. 2017;13:201-6.

19. Bluth K, Campo RA, Futch WS, Gaylord SA. Age and gender differences in the associations of self-compassion and emotional well-being in a large adolescent sample. J Youth Adolesc. 2017;46:840-53.

20. Schieman S, Van Gundy K. The personal and social links between age and selfreported empathy. Soc Psychol Q. 2000;63:152.

21. Maylor EA, Moulson JM, Muncer AM, Taylor LA. Does performance on theory of mind tasks decline in old age? Br J Psychol. 2002;93:465-85.

22. Bailey PE, Henry JD, Von, Hippel W. Empathy and social functioning in late adulthood. Aging Ment. Health. 2008;12:499-503.

23. Bailey $P E$, Henry JD. Growing less empathic with age: disinhibition of the selfperspective. J Gerontol B Psychol Sci Soc Sci. 2008;63:P219-p226.

24. Beadle JN, Paradiso S, Kovach C, Polgreen L, Denburg NL, Tranel D. Effects of agerelated differences in empathy on social economic decision-making. Int Psychogeriatr. 2012;24:822-33.

25. Beadle JN, Sheehan AH, Dahlben B, Gutchess AH. Aging, empathy, and prosociality. J Gerontol B Psychol Sci Soc Sci. 2015;70:215-24.

26. Chen YC, Chen CC, Decety J, Cheng Y. Aging is associated with changes in the neural circuits underlying empathy. Neurobiol Aging. 2014;35:827-36.

27. Wieck C, Kunzmann U. Age differences in empathy: multidirectional and contextdependent. Psychol Aging. 2015;30:407-19.

28. Bailey PE, Brady B, Ebner NC, Ruffman T. Effects of age on emotion regulation, emotional empathy, and prosocial behavior. J Gerontol B Psychol Sci Soc Sci. 2020;75:802-10.

29. Oh J, Chopik W, Konrath S, Grimm K. Longitudinal changes in empathy across the life span in six samples of human development. Soc Psychol Person Sci. 2019;11:194855061984942.

30. Helson R, Jones C, Kwan VS. Personality change over 40 years of adulthood: hierarchical linear modeling analyses of two longitudinal samples. J Pers Soc Psychol. 2002;83:752-66.

31. Grühn D, Rebucal K, Diehl M, Lumley M, Labouvie-Vief G. Empathy across the adult lifespan: Longitudinal and experience-sampling findings. Emotion. 2008;8:753-65. 
32. Hamilton WD. The moulding of senescence by natural selection. J Theor Biol. 1966;12:12-45.

33. Foster EA, Franks DW, Mazzi S, Darden SK, Balcomb KC, Ford JK, et al. Adaptive prolonged postreproductive life span in killer whales. Science. 2012;337:1313-1313.

34. Holt-Lunstad J, Smith TB, Baker M, Harris T, Stephenson D. Loneliness and social isolation as risk factors for mortality: a meta-analytic review. Perspect Psychol Sci. 2015;10:227-37.

35. Lee EE, Depp C, Palmer BW, Glorioso D, Daly R, Liu J, et al. High prevalence and adverse health effects of loneliness in community-dwelling adults across the lifespan: role of wisdom as a protective factor. Int Psychogeriatr. 2019;31:1447-62.

36. Cacioppo J, Patrick W. Loneliness: human nature and the need for social connection. New York: W.W. Norton \& Company, Inc.; 2008.

37. Woolf $\mathrm{SH}$, Schoomaker $\mathrm{H}$. Life expectancy and mortality rates in the United States, 1959-2017. Jama. 2019;322:1996-2016.

38. Jeste DV, Di Somma S, Lee EE, Nguyen TT, Scalcione M, Biaggi A, et al. Study of loneliness and wisdom in 482 middle-aged and oldest-old adults: a comparison between people in Cilento, Italy and San Diego, USA. Aging Ment Health. 2020:1-11.

39. Nguyen TT, Lee EE, Daly RE, Wu TC, Tang Y, Tu X, et al. Predictors of loneliness by age decade: study of psychological and environmental factors in 2,843 community-dwelling americans aged 20-69 years. J Clin Psychiatry. 2020;81.

40. Jeste DV, Thomas ML, Liu J, Daly RE, Tu XM, Treichler E, et al. Is spirituality a component of wisdom? Study of 1,786 adults using expanded San Diego Wisdom Scale (Jeste-Thomas Wisdom Index). J Psychiatr Res. 2021;132:174-81.

41. Grennan G, Balasubramani PP, Alim F, Zafar-Khan M, Lee EE, Jeste DV. et al. Cognitive and neural correlates of loneliness and wisdom during emotion processing across the adult lifespan. Cerebral Cortex. 2021;31:3311:22.

42. Nguyen TT, Zhang X, Wu TC, Liu J, Le C, Tu XM, et al. Association of loneliness and wisdom with gut microbial diversity and composition: an exploratory study. Front Psychiatry. 2021;12:648475.

43. Ardelt M. Antecedents and effects of wisdom in old age: a longitudinal perspective on aging well. Res Aging. 2000;22:360-94.

44. Ardelt M, Gerlach KR, Vaillant GE. Early and midlife predictors of wisdom and subjective well-being in old age. J Gerontol B Psychol Sci Soc Sci. 2018;73:1514-25.

45. Van Patten R, Lee EE, Daly R, Twamley E, Tu XM, Jeste DV. Assessment of 3dimensional wisdom in schizophrenia: Associations with neuropsychological functions and physical and mental health. Schizophr Res. 2019;208:360-9.

46. Heym N, Firth J, Kibowski F, Sumich A, Egan V, Bloxsom C. Empathy at the heart of darkness: empathy deficits that bind the dark triad and those that mediate indirect relational aggression. Front Psychiatry. 2019;10:95.

47. Friis AM, Johnson MH, Cutfield RG, Consedine NS. Kindness matters: a randomized controlled trial of a mindful self-compassion intervention improves depression, distress, and $\mathrm{HbA1c}$ among patients with diabetes. Diabetes Care. 2016;39:1963-71.

48. Sommers-Spijkerman MPJ, Trompetter HR, Schreurs KMG, Bohlmeijer ET. Compassion-focused therapy as guided self-help for enhancing public mental health: a randomized controlled trial. J Consult Clin Psychol. 2018;86:101-15.

49. Mantzios M, Wilson JC. Making concrete construals mindful: a novel approach for developing mindfulness and self-compassion to assist weight loss. Psychol Health. 2014;29:422-41.

50. Yadavaia JE, Hayes SC, Vilardaga R. Using acceptance and commitment therapy to increase self-compassion: a randomized controlled trial. JContextual Behav Sci. 2014;3:248-57.

51. Wallmark E, Safarzadeh K, Daukantaite D, Maddux RE. Promoting altruism through meditation: an 8-week randomized controlled pilot study. Mindfulness. 2013;4:223-34.

52. Larti N, Ashouri E, Aarabi A. The effect of an empathy role-play program for operating room nursing students. J Educ Evaluation Health Prof. 2018;15:29.

53. de Bruin El, van der Zwan JE, Bögels SM. A RCT comparing daily mindfulness meditations, biofeedback exercises, and daily physical exercise on attention control, executive functioning, mindful awareness, self-compassion, and worrying in stressed young adults. Mindfulness. 2016;7:1182-92.

54. Braehler C, Gumley A, Harper J, Wallace S, Norrie J, Gilbert P. Exploring change processes in compassion focused therapy in psychosis: results of a feasibility randomized controlled trial. Br J Clin Psychol. 2013;52:199-214.

55. Friis AM, Johnson MH, Cutfield RG, Consedine NS. Does kindness matter? Selfcompassion buffers the negative impact of diabetes-distress on HbA1c. Diabet Med. 2015;32:1634-40.

56. Jeste DV, Savla GN, Thompson WK, Vahia IV, Glorioso DK, Martin AS, et al. Association between older age and more successful aging: critical role of resilience and depression. Am J Psychiatry. 2013;170:188-96.

57. Thomas ML, Kaufmann CN, Palmer BW, Depp CA, Martin AS, Glorioso DK, et al. Paradoxical trend for improvement in mental health with aging: a community-based study of 1,546 adults aged 21-100 years. J Clin Psychiatry. 2016;77:e1019-1025.

58. Kroenke K, Spitzer RL, Williams JB. The PHQ-9: validity of a brief depression severity measure. J Gen Intern Med. 2001;16:606-13.

59. Derogatis LR, Melisaratos N. The Brief Symptom Inventory: an introductory report. Psychol Med. 1983;13:595-605.

60. Cohen S, Kamarck T, Mermelstein R. A global measure of perceived stress. J Health Soc Behav. 1983;24:385-96.

61. Connor KM, Davidson JR. Development of a new resilience scale: the ConnorDavidson Resilience Scale (CD-RISC). Depress Anxiety. 2003;18:76-82.

62. Scheier MF, Carver CS, Bridges MW. Distinguishing optimism from neuroticism (and trait anxiety, self-mastery, and self-esteem): a reevaluation of the Life Orientation Test. J Personal Soc Psychol. 1994;67:1063-78.

63. Diener E, Emmons RA, Larsen RJ, Griffin S. The satisfaction with life scale. J Pers Assess. 1985;49:71-75.

64. Russell DW. UCLA Loneliness Scale (Version 3): reliability, validity, and factor structure. J Pers Assess. 1996;66:20-40.

65. Ware JE Jr., Sherbourne CD. The MOS 36-item short-form health survey (SF-36). I. Conceptual framework and item selection. Med Care. 1992;30:473-83.

66. Raes F, Pommier E, Neff KD, Van, Gucht D. Construction and factorial validation of a short form of the Self-Compassion Scale. Clin Psychol Psychother. 2011;18:250-5

67. Field A, Miles J, Field Z. Discovering statistics using R. London: Sage Publications; 2012.

68. Tang W, Tu XM (eds). Modern clinical trial analysis. New York: Springer Science; 2012.

69. Pan W. Akaike's information criterion in generalized estimating equations. Biometrics. 2001;57:120-5.

70. Eisenberg N, Cumberland A, Guthrie IK, Murphy BC, Shepard SA. Age changes in prosocial responding and moral reasoning in adolescence and early adulthood. J Res Adolesc. 2005;15:235-60.

71. Allemand $M$, Gruenenfelder-Steiger A, Fend $H$. Empathy development in adolescence predicts social competencies in adulthood. J Personality. 2015;83:229-41.

72. Davis MH, Franzoi SL. Stability and change in adolescent self-consciousness and empathy. J Res Personal. 1991;25:70-87.

73. Van der Graaff J, Branje S, De Wied M, Hawk S, Van Lier P, Meeus W. Perspective taking and empathic concern in adolescence: gender differences in developmental changes. Dev Psychol. 2014;50:881-8.

74. O'Brien E, Konrath SH, Gruhn D, Hagen AL. Empathic concern and perspective taking: linear and quadratic effects of age across the adult life span. J Gerontol B Psychol Sci Soc Sci. 2013;68:168-75.

75. Nakagawa S, Takeuchi $H$, Taki $Y$, Nouchi R, Sekiguchi A, Kotozaki $Y$, et al. White matter structures associated with loneliness in young adults. Sci Rep. 2015;5:17001.

76. Zhang S, Zhang Y, Yuan B. Mediating effect of self-esteem and empathy on the relationship between loneliness and cyber-bulling in middle and high school students in Liaoning Province. J Hyg Res. 2019;48:446-57.

77. Davis M. Measuring individual differences in empathy: evidence for a multidimensional approach. J Personalilty Soc Psychol. 1983;44:113-26.

78. Eisenberg N, Lennon R. Sex differences in empathy and related capacities. Psychological Bull. 1983;94:100-31.

79. Hajibabaee F, M AF, Ameri Z, Salehi T, Hosseini F. The relationship between empathy and emotional intelligence among Iranian nursing students. Int J Med Educ. 2018;9:239-43.

80. Pommier E, Neff KD, Tóth-Király I. The development and validation of the compassion scale. Assessment. 2019;27:21-39.

81. Burnell L, Agan D. Compassionate care: can it be defined and measured? the development of the compassionate care assessment tool. Int J Caring Sci. 2013;6:180-7.

82. Mercadillo RE, Diaz JL, Pasaye EH, Barrios FA. Perception of suffering and compassion experience: brain gender disparities. Brain Cogn. 2011;76:5-14.

83. Stellar JE, Manzo VM, Kraus MW, Keltner D. Class and compassion: socioeconomic factors predict responses to suffering. Emotion. 2012;12:449-59.

84. Lopez A, Sanderman R, Ranchor AV, Schroevers MJ. Compassion for others and self-compassion: levels, correlates, and relationship with psychological wellbeing. Mindfulness. 2018;9:325-31.

85. Li T, Siu PM. Socioeconomic status moderates age differences in empathic concern. J Gerontol B Psychol Sci Soc Sci. 2021;76:507-17.

86. Kraus MW, Côté S, Keltner D. Social class, contextualism, and empathic accuracy. Psychol Sci. 2010;21:1716-23.

87. Saarinen Al, Keltner D, Dobewall H, Lehtimäki T, Keltikangas-Järvinen L, Hintsanen $M$. The relationship of socioeconomic status in childhood and adulthood with compassion: a study with a prospective 32-year follow-up. PLOS ONE. 2021;16: e0248226.

88. Durkin M, Beaumont E, Hollins Martin CJ, Carson J. A pilot study exploring the relationship between self-compassion, self-judgement, self-kindness, compassion, 
professional quality of life and wellbeing among UK community nurses. Nurse Educ Today. 2016;46:109-14.

89. Neff K, Pommier E. The relationship between self-compassion and other-focused concern among college undergraduates, community adults, and practicing meditators. Self Identity. 2012;12:1-17.

90. Kirby JN, Tellegen CL, Steindl SR. A meta-analysis of compassion-based interventions: current state of knowledge and future directions. Behav Ther. 2017;48:778-92.

91. Morgan TL, Semenchuk BN, Ceccarelli L, Kullman SM, Neilson CJ, Kehler DS, et al. Self-compassion, adaptive reactions and health behaviours among adults with prediabetes and type 1, type 2 and gestational diabetes: a scoping review. Can J Diabetes. 2020;44:555-565. e552

92. Biber DD, Ellis R. The effect of self-compassion on the self-regulation of health behaviors: a systematic review. J Health Psychol. 2019;24:2060-71.

93. Paolillo EW, Sun-Suslow N, Pasipanodya EC, Morgan EE, Ellis RJ, Jeste DV, et al. Pre-frailty predicts cognitive decline at 2-year follow-up in persons living with HIV. J Neurovirol. 2020;26:168-80.

94. Morlett Paredes A, Lee EE, Chik L, Gupta S, Palmer BW, Palinkas LA, et al. Qualitative study of loneliness in a senior housing community: the importance of wisdom and other coping strategies. Aging Ment Health. 2021;25:559-66.

95. Jeste DV, Lee EE, Cacioppo S. Battling the modern behavioral epidemic of loneliness: suggestions for research and interventions. JAMA Psychiatry 2020.

96. López J, Perez-Rojo G, Noriega C, Carretero I, Velasco C, Martinez-Huertas JA, et al. Psychological well-being among older adults during the COVID-19 outbreak: a comparative study of the young-old and the old-old adults. Int Psychogeriatr. 2020;32:1365-70.

\section{ACKNOWLEDGEMENTS}

This study was supported, in part, by the National Institutes of Health [NIMH R01MH094151 (PI: Dilip V. Jeste), NIMH K23MH119375-01 (PI: Ellen E. Lee), National Institutes of Health Grant NIH UL1TR001442 of CTSA (PI: Gary Firestein, MD)], by the VA San Diego Healthcare System, and by the Stein Institute for Research on Aging (Director: Dilip V. Jeste, MD) at the University of California San Diego. The authors have no conflicts of interest with the work described. The content of this paper is solely the responsibility of the authors and does not necessarily represent the official views of the $\mathrm{NIH}$.

\section{COMPETING INTERESTS}

The authors declare no competing interests.

\section{ADDITIONAL INFORMATION}

Correspondence and requests for materials should be addressed to E.E.L.

Reprints and permission information is available at http://www.nature.com/ reprints

Publisher's note Springer Nature remains neutral with regard to jurisdictional claims in published maps and institutional affiliations.

(i) Open Access This article is licensed under a Creative Commons Attribution 4.0 International License, which permits use, sharing, adaptation, distribution and reproduction in any medium or format, as long as you give appropriate credit to the original author(s) and the source, provide a link to the Creative Commons license, and indicate if changes were made. The images or other third party material in this article are included in the article's Creative Commons license, unless indicated otherwise in a credit line to the material. If material is not included in the article's Creative Commons license and your intended use is not permitted by statutory regulation or exceeds the permitted use, you will need to obtain permission directly from the copyright holder. To view a copy of this license, visit http://creativecommons. org/licenses/by/4.0/.

(c) The Author(s) 2021 